Proc. Indian Acad. Sci. (Earth Planet. Sci.), Vol. 98, No. 3, October 1989, pp. 297-308.

C. Printed in India.

\title{
Generation of low-frequency oscillations in the subsolar venus ionopause
}

\author{
R N SINGH and HARI OM UPADHYAY \\ Department of Applied Physics, Institute of Technology, Banaras Hindu University, Varanasi \\ 221005, India \\ MS received 31 August 1987; revised 18 February 1989
}

\begin{abstract}
The steep electron density gradient, the magnetic field curvature and the effect of gravity make the Venus ionopause region unstable. The onset of flute instability in the Venus ionopause plasma has been studied. The dispersion of waves and their growth have been studied using in situ measured data. The existence of low frequency waves in the subsolar magnetic field data ionopause region has been interpreted to arise as the result of flute instability.
\end{abstract}

Keywords. Venus ionopause oscillations; ionopause instability; flute instability; intarchange instability.

\section{Introduction}

Although the interaction of solar wind interaction with terrestrial magnetosphere is known to result in various types of low frequency perturbations, its interaction with Venus magnetospheric plasma is somewhat different due to lack of ambient magnetic field of Venus. The solar wind magnetic field is frozen-in and moves with the solar wind into the Venus magnetosphere. The motional electric field in the solar wind is given by $\mathbf{E}+\mathbf{V} \times \mathbf{B}=0$ which implies $\mathbf{E} \cdot \mathbf{B}=0$. The $\mathbf{E}$ and $\mathbf{B}$ fields are directed in different directions. In a frozen-in plasma the $\mathbf{E}$ and $\mathbf{B}$ field instabilities are excited. These instabilities are governed by different physical parameters in different regions of the plasma. In this paper, we consider the instability of a magnetic field in the ionopause region. The oncoming solar wind is mass-loaded and is consequently slowed down as it approaches the denser ionosphere of Venus. The magnetic field lines and the solar wind plasma drape around the planet and a well-defined magnetic field and electron density gradients are established in the subsolar region of the Venus ionopause. The gravitational force acting on the stratified magnetoplasma of the Venus is known to provide stabilizing effects. However, the electron density and magnetic field gradients in the subsolar region of the Venus ionopause are known to change significantly from time to time which make the subsolar region susceptible to various types of instabilities. The recorded magnetic field data (OMAG) on board the Pioneer Venus Orbiter have been shown to exhibit significant magnetic field oscillations (Russell et al 1987).

The electron density gradient and the gravitational force in certain regions of the ionopause region are directed in opposite directions. As the electron density gradient in the ionopause region increases the magnetic field curvature also increases. These changes, in addition to piling-up of the magnetic field, are important for the onset of interchange or flute instability in the ionopause region of Venus (Rosenbluth et al 
1962; Kadomtsev 1965; Post 1969). In the normal conditions of Venus magnetospheric plasma, the curvature of the magnetic field lines is small (large radius of curvature) and the gravitational force is inadequate to destabilize the subsolar Venus ionopause region. However, in the case of enhanced solar wind dynamic pressure, the mass loading of the solar wind and the draping of the magnetic field lines around the planet becomes more pronounced. The draping results in a significant increase in the curvature of magnetic field lines. The existence of suitable electron density gradient is important for exciting the flute instability. We show that the enhanced curvature of the magnetic field lines in the Venus subsolar ionopause develops an additional force in the direction of the gravitational force which acts like a fictitious gravitational force and triggers flute instability. The dispersion properties of these waves and their growth/decay processes have been studied. The low frequency oscillations seen in the Venus magnetic field records are attributed to flute instability.

\section{Dispersion equation}

We consider the subsolar Venus ionopause region with plasma density gradient along the $x$-axis with magnetic field directed along the $z$-axis. The $y$-axis completes the orthogonal co-ordinate system as shown in figure 1. The ionopause with finite plasma density gradient and embedded in the magnetic field conforms to the frozen-in condition for which $\mathbf{E}+(\mathbf{V} \cdot \mathbf{B})=0$ and $\mathbf{E} \cdot \mathbf{B}=0$. The electrons and ions drift parallel to the $y$-axis with velocity

$$
\left(\mathbf{v}_{0}\right)_{e, i}=\mathbf{E} \cdot \mathbf{B} / \mathbf{B}^{2}
$$

In the equilibrium state, the electric field is small, the electron drift becomes negligible

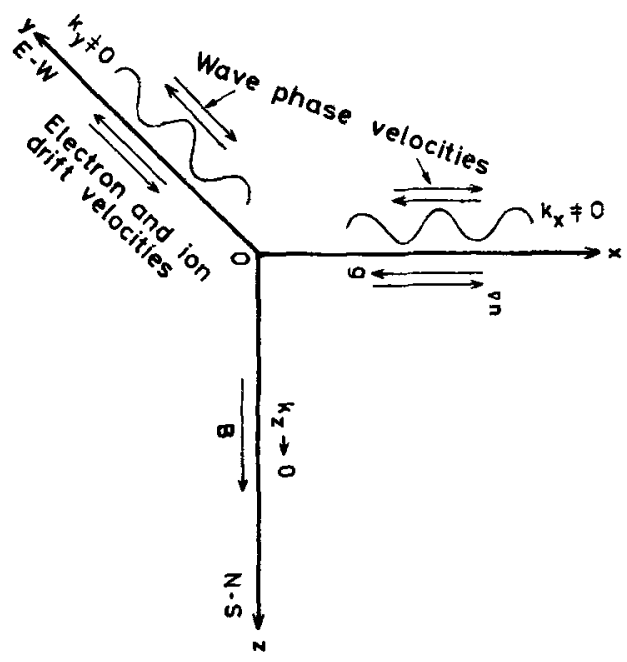

Figure 1. Co-ordinate system, configuration of plasma gradient, magnetic field, charged particle drifts atnd wave propagation. 
and the ions drift along the $x$-axis with a velocity

$$
\mathrm{v}_{0 i}=\mathrm{g} / \Omega_{i},
$$

where $\mathbf{g}$ is the acceleration due to gravity of Venus. The differential electron and ion drift velocities in the ionopause region excite the plasma perturbations in the plasma density-gradient region. Considering two-dimensional perturbations in the $x-y$ plane to be of the form $\exp (-i \omega t+\mathbf{k} \cdot \mathbf{r})$, the dispersion equation for interchange or flute oscillations has been derived by many workers.

During the formation of Venus ionopause, the electron flux is swept by the oncoming solar wind and a region with minimum gradient of electron density is formed. However, in this region, the microscopic charge density neutrality is disturbed and gives rise to self-consistent electric field in this region. For the formation of well-developed ionopause in the Venus the condition $\mathbf{E}+\left(\mathbf{V}_{s w} \times \mathbf{B}\right)=0$ which also implies $\mathbf{E} \cdot \mathbf{B}=0$. To consider the hydrodynamic drift the contribution of the term $\left[\left(\mathbf{g} \times \mathbf{B} / \Omega_{i} B\right) \nabla \mathbf{E}\right]$ is negligible; therefore for this problem, the dispersion equation for hydrodynamic oscillations obtained by the Kadomtsev (1965) reduces to that obtained by Post (1969). Accounting for the Doppler-shift for ions only, the dispersion equation, in the co-ordinate system (figure 1), was obtained (Post 1969). Although this equation was somewhat different from that given by Kadomtsev (1965), the computational results neither show up much difference nor alter the physical implications of the results obtained using Post (1969). The dispersion equation is written as

$$
\omega^{2}\left[1+\left(V_{A}^{2} / C^{2}\right)\right]-\left[\left(g k_{y} / \Omega_{i}\right)\left(V_{A}^{2} / C^{2}\right) \omega\right]-a g\left(k_{y} / k\right)^{2}=0
$$

where $V_{A}, \Omega_{i}$ and $a=\left(1 / n_{0}\right)\left(\mathrm{d} n_{0} / \mathrm{d} x\right)$ are Alfven velocity, ion cyclotron frequency and plasma inhomogeneity inverse scale length respectively. The wave vector in the $x-y$ plane $k=\left(k_{x}^{2}+\dot{k}_{y}^{2}\right)^{1 / 2}$. The motion of ions in this region is balanced by the gravitational and the magnetic curvature forces. For a given velocity and mass of ions, the term corresponding to $\left(\Omega_{e}\right)^{-1}$ is smaller than that containing $\left(\Omega_{i}\right)^{-1}$.

$$
m_{i} g=m_{i} v_{i}^{2} / R \text { and } \rho_{i}=v_{i} / \Omega_{i}
$$

where $R$ is the radius of curvature of the magnetic field lines and $\rho_{i}$ is the ion gyroradius. From these equations one obtains an expression for fictitious gravitational acceleration

$$
\mathbf{g}=\rho_{i}^{2} \Omega_{i}^{2} / R
$$

$R$ also conforms to the curvature of the steady state subsolar plasma. It is obvious from (5) that the combined effect of the three parameters replaces the gravitational acceleration of Venus by a fictitious gravitational acceleration. Substituting for $g$ in (3), the dispersion equation for $V_{A} \ll C$ is rewritten as

$$
\omega^{2}-\left(k_{y} R\left(\rho_{i} / R\right)^{2} \frac{V_{A}^{2}}{C^{2}}\right) \Omega_{i} \omega-a R\left(\rho_{i} / R\right)^{2}\left(k_{y} / k\right)^{2} \Omega_{i}^{2}=0 .
$$


The solution of this equation is

$$
\begin{aligned}
\omega= & \frac{\Omega_{i}}{2}\left(k_{y} R \frac{\rho_{i}^{2}}{R^{2}} \frac{V_{A}^{2}}{C^{2}}\right) \\
& \pm\left\{\left[k_{y} R\left(\rho_{i} / R\right)^{2} \frac{V_{A}^{2}}{C^{2}}\right]^{2}+4 a R\left(\rho_{i} / R\right)^{2}\left(k_{y} / k\right)^{2}\right\}^{1 / 2} \cdot \frac{\Omega_{i}}{2} .
\end{aligned}
$$

If the second term in the curly bracket is small, the low frequency perturbations are stable and the frequency of these oscillations is given by

$$
\omega=\Omega_{i} k_{y} R\left(\rho_{i} / R\right)^{2}\left(V_{A}^{2} / C^{2}\right)
$$

In the case of enhanced solar wind dynamic pressure and mass-loading $R$ decreases which may increase the second term satisfying the inequality $a R<0$. In such conditions the solution of $(6)$ for real $k$ values becomes complex $\omega=\omega_{r} \pm i \gamma\left(\gamma \ll(\omega)_{r}\right)$. Since plane wave perturbations are defined as $\exp (-i \omega t+\mathbf{k r})$, the positive imaginary component gives the growth rate and under the approximation of $k_{x}=0, k_{y} \simeq k$ is written as

$$
\gamma=\left(\rho_{i} / R\right) \Omega_{i} \sqrt{a R} \mathrm{~s}^{-1} .
$$

We find that the oscillation frequencies are governed by $R$ in the ionopause region and on the ratio of $V_{A} / C$ which depends predominantly on the mass loading of the solar wind. We show in this paper how the growth rate of these oscillations depends on the plasma density gradient and $R$.

\section{Magnetic field observations}

The magnetometer onboard PVO measures the three components of the Venus magnetic field and the resultant total magnetic field during the entire orbit around Venus (Russell et al 1980). During each orbit, the high resolution magnetic field data are recorded for two hours around the periapsis in addition to normal mode recording during the entire orbit. Thus, magnetic field data consist of high resolution data in the near space and the normal mode data for the distant space around Venus. This study concerns the nature of magnetic field perturbations in the subsolar region of the Venus ionopause dominated by the plasma density gradient, the magnetic field curvature and its gradient. Two types of magnetic field structures are commonly observed in the subsolar ionopause region. In the ionopause region dominated by plasma convection and magnetic diffusivity, a magnetic structure is commonly observed near periapsis, in which the magnetic field penetrates forming a bay and under suitable condition grows and forms a belt-like structure around the periapsis (Luhmann et al 1984; Cravens et al 1984 and Phillips et al 1984). Another commonly observed magnetic field variation shows the piling up of the magnetic field around lower SZA periapsis forming a smoothly varying hump-like structure. Like the bay structure at the periapsis, the hump structure also shows, at times, large period magnetic field perturbations. Typical examples of bay and humped magnetic structures with and without oscillations are shown in figures $2(a, b)$. In addition to long-period magnetic oscillations around the periapsis, smaller period oscillations are also seen 

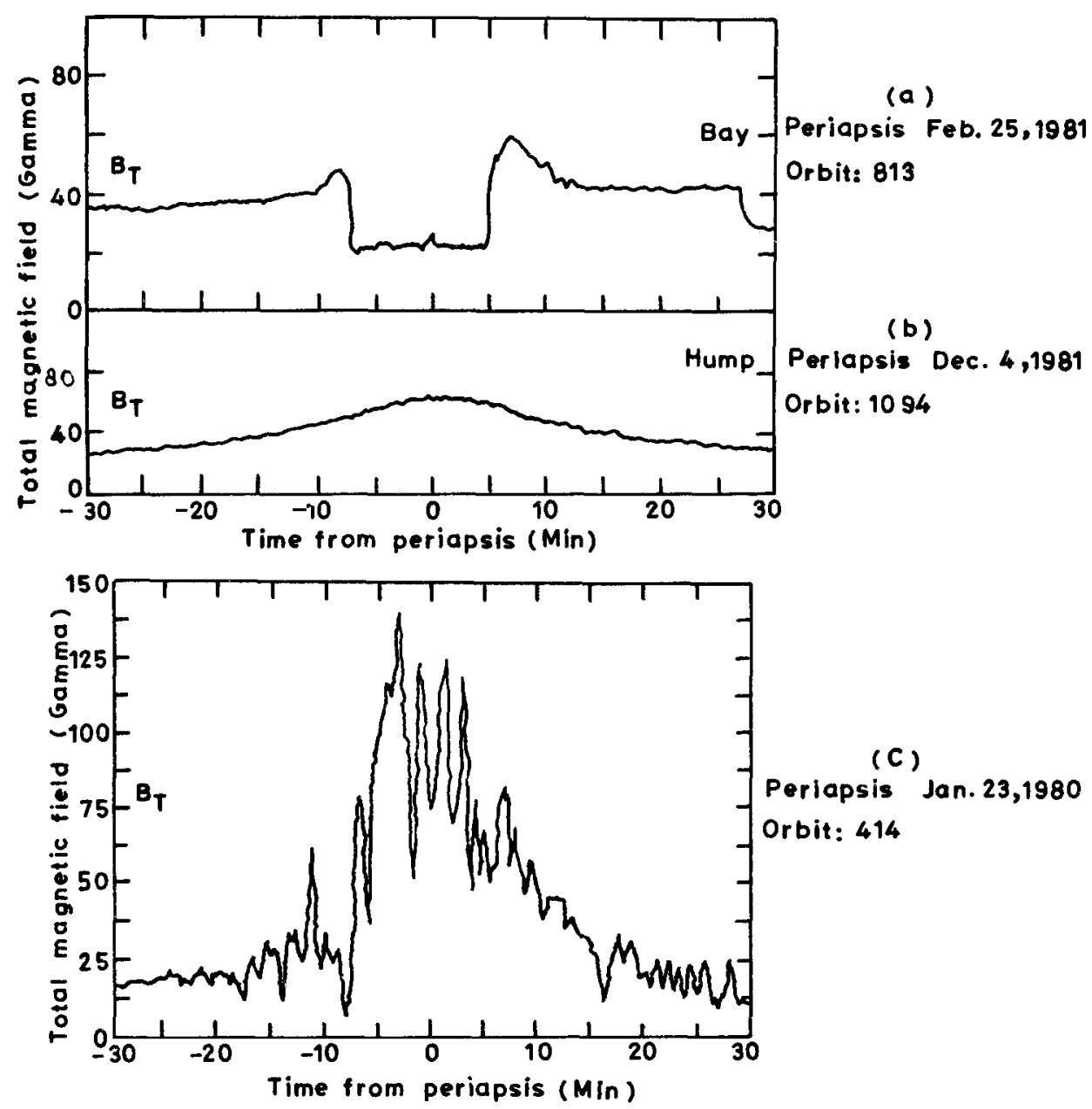

Figure 2. Total magnetic field variation near subsolar periapsis: (a) Bay structure, (b) Hump structure, (c) Large amplitude oscillations.

at higher solar zenith angies. The long-period and large amplitude oscillations are generally seen with large amplitude east-west magnetic field component in the periapsis region. With increasing solar zenith angles the period as well as the amplitude of these oscillations are found to change. In the pre-terminator and trans-terminator regions, these oscillations appear to be more complex arising from the superposition of oscillations produced by more than one process.

The subsolar region of the ionopause around the periapsis is stagnant and the flow velocity along the field lines is rather small which increases with increasing solar zenith angles. The velocity shear in this region is almost non-existent and is not adequate for creating favourable conditions for the onset of Kelvin-Helmholtz instability (Elphic and Ershkovich 1984). However, during the incidence of solar wind with enhanced dynamic pressure, the density gradient in the ionopause increases. The cnhanced, mass-loading of the solar wind reduces the radius of curvature of the ionopause and thereby enhances the fictitious gravitational force of the Venus. Under the influence of density gradient and the fictitious gravitational force the ionopause 
surface under suitable conditions becomes unstable to interchange forces giving rise to flute instability. This instability mechanism gives rise to large amplitude oscillations. Examples of such oscillations are shown in figure 2(c). The $\Delta B^{\prime} B$ of these oscillations are seen to vary from 0.1 to 0.8 and the period of these oscillations varies from 100 to 1000 seconds.

PVO orbits with enhanced altitude of periapsis, at times, are found to be closer to Venus bow shock. In such cases shock-generated magnetic field oscillations are also seen to exist. These oscillations decrease with increasing distance from the shock. These oscillations at the bow shock are correlated with well-defined oscillations at the periapsis. Such observations being limited in number, it is difficult to find the causative role of these oscillations in structuring the periapsis magnetic field.

\section{Results and discussion}

The flute mode oscillations may be excited by differential electron and ion drifts and are controlled by various plasma and field parameters in the Venus ionopause region. The gyroradius of ions and the curvature of the frozen-in magnetic field in the ambient plasma of Venus ionopause are two important parameters governing the oscillation frequencies and onset of the flute instability. Using OMAG, we have computed the gyroradius and $R$ for $\mathrm{O}^{+}$ions in the energy range of 0.1 to $30 \mathrm{eV}$. Variations of $\rho_{i}$ and $R$ with velocity of $\mathrm{O}^{+}$ions for different values of magnetic field are shown in figure 3. The corresponding energy of $\mathrm{O}^{+}$ions is shown in the upper axis. Although these variations follow from the simple relation given in (4), the variations of these

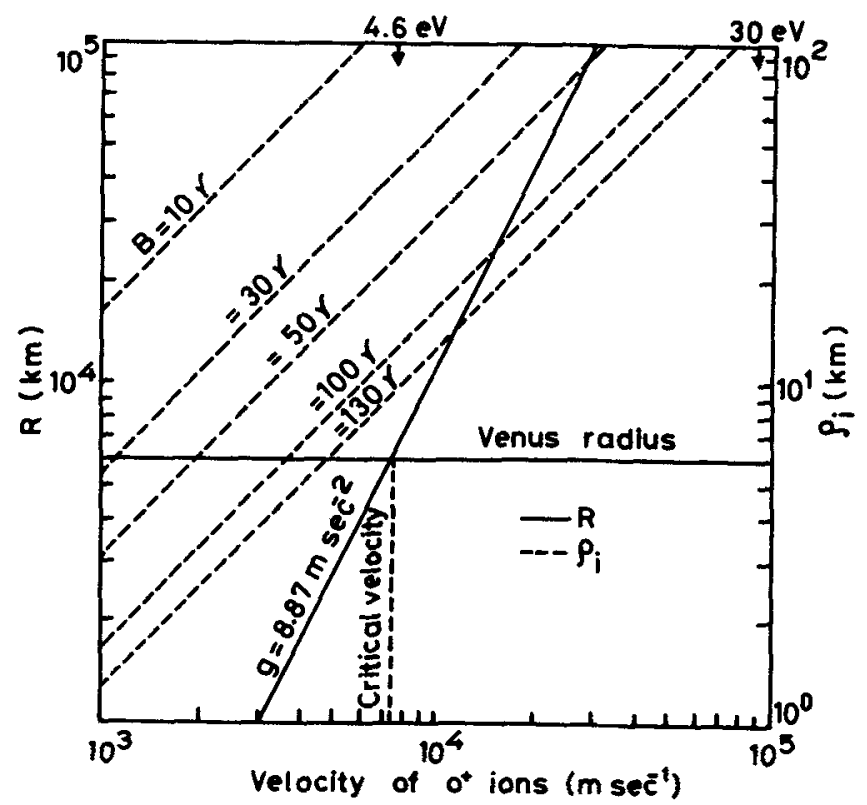

Figure 3. Variation of radius of gyration of ions with velocity of ions for different Venus magnetic fields. 
parameters for various values of measured magnetic field are shown for clarity. The solid curves show the value of $R$ corresponding to Venus gravitational acceleration of $8.87 \mathrm{~m} \mathrm{~s}^{-2}$. The value of $R$ of the ionopause boundary (same as the magnetic field curvature under frozen-in condition) changes considerably with enhanced massloading of solar wind and corresponds to an enhanced gravitational force and an associated increase in the plasma density gradient. These two parameters alter the boundary conditions at the Venus subsolar ionopause. In terms of plasma pressure difference and mass density at the ionopause, $R$ is expressed as

$$
R=\frac{2\left(p_{o}-p_{i}\right)}{\left(d_{i}+d_{o}\right) g}
$$

where $p_{o}$ and $p_{i}$ refer to outer and inner plasma pressures with reference to the ionopause boundary and $d_{i}, d_{o}$ are the inner and outer mass densities. Using $R$, the field and the plasma parameters, we have computed $\omega$ variations with $k$ values for these waves (figure 4). Various sets of curves are shown for a range of $V_{A} / C$ values which are known to change with changing solar wind dynamic pressure and the mass-loading of the solar wind. The variation of $V_{A} / C$ has a bearing on the wide

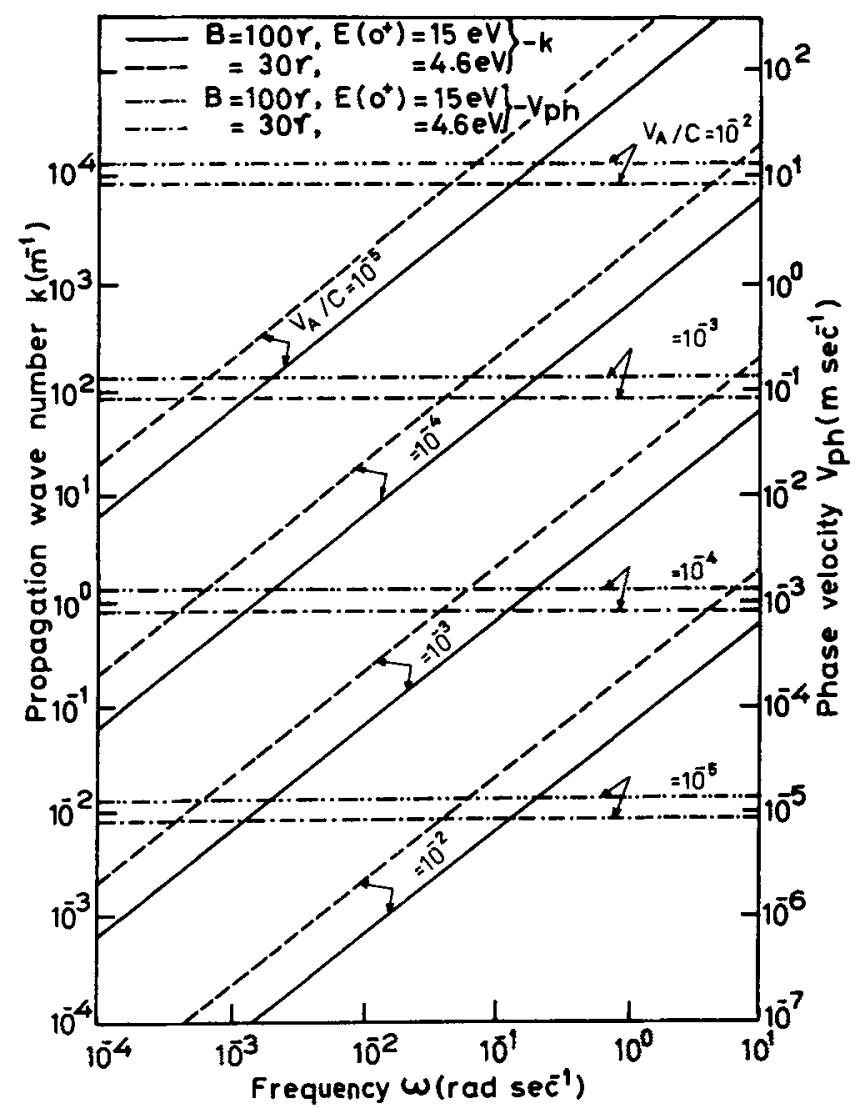

Figure 4. The wave vector $k$ and phase velocity variation with $\omega$ for different values of normalized Alfven velocity $V_{A} / C$. 

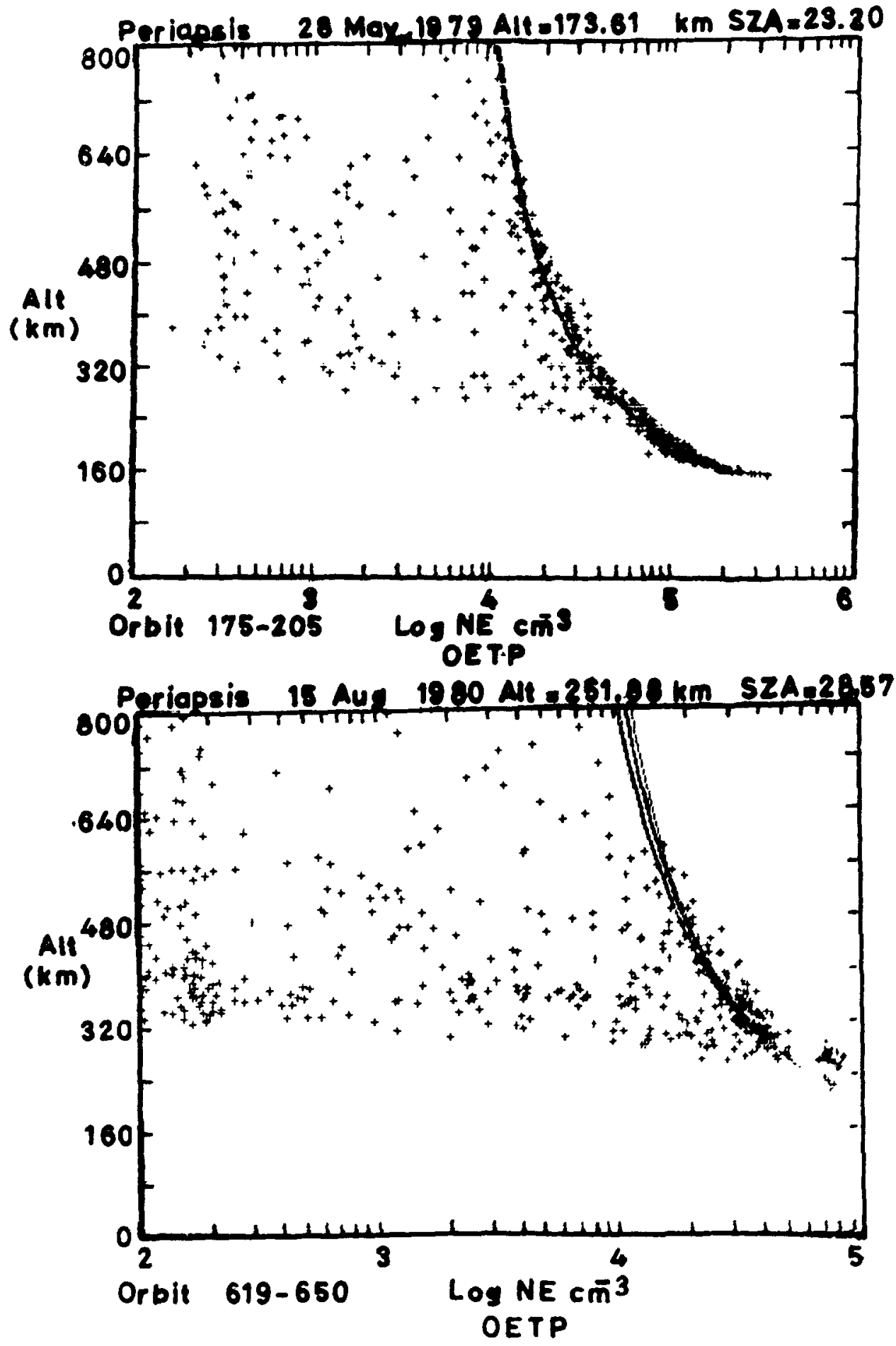

Figure 5. Electron density variations with altitude showing well-defined lower boundary of the subsolar ionosphere. (a) Ne versus altitude plot for orbits 175--205. (b) Ne versus altitude plot for orbits 619-650. 
range of plasma density and magnetic field variations in the periapsis region. The horizontal set of lines shows the phase velocity of these oscillations. Figures $5(\mathrm{a}, \mathrm{b})$ show the electron density profiles for low SZA ( $25^{\circ}$ around Sun-Venus line) orbits for two seasons covering orbits 175-205 and 619-650. The scatter plots of electron density do not provide any idea of the required density gradient which appears corresponding to the lower periapsis altitudes. The ion densities corresponding to the lowest and highest values of electron density seen from figures $5(\mathrm{a}, \mathrm{b})$ have been used to compute $V_{A}$ and setting the $V_{A} / C$ parameters in this analysis. The corresponding phase velocity variations for different values of measured magnetic field are shown in figure 4. For lower values of $V_{A} / C$, the phase velocities of these waves are fairly small. Even for $V_{A} / C=10^{-2}$, the phase velocity of these waves is of the order of tens of meters per second. For spacecrafts moving with a velocity of approximately $10 \mathrm{~km} \mathrm{~s}^{-1}$ these oscillations appear almost stationary. Under favourable conditions, the spacecraft may sample several wavelengths of these oscillations. Examples of 1-5 wavelengths of magnetic field oscillations are often seen in the magnetic records. The number of wavelengths is limited by relative strengths of other components of magnetic field, the thickness of the ionopause layer altitude of the periapsis and the direction of the spacecraft movement near the periapsis.

The magnetic field oscillations away from the low SZA periapsis are also seen at times. The period of these oscillations is found to decrease with increasing SZA and the amplitude of these oscillations also decreases with increasing SZA values. The Kelvin-Helmholtz mechanism has been studied and it is believed that the shearing flow is rather small for low SZA and becomes dominant in the higher SZA regions of Venus ionopause. The shearing flow near the terminator region does provide favourable conditions for the onset of the K-H instability (Elphic and Ershkovich 1984). The study of flute instability shows that the pressure difference and the effect of mass-loading which control the curvature of the Venus ionopause boundary and drive the flute instability decrease with increasing SZA. The magnetic field oscillations corresponding to higher SZA in the Venus pre- and post-terminator regions show a hybrid type of oscillation which seem to be affected either by local plasma fluctuations or by some other instability mechanism. The velocity shear and the viscosity of the streaming solar wind plasma start playing a dominant role and the $\mathrm{K}-\mathrm{H}$ instability becomes comparatively a more appropriate mechanism to excite these oscillations in the pre- and the post-terminator region (Brace et al 1983).

The feasibility of excitation of the flute instability driven oscillations and their growth rate using the Venus ionopause parameters has been studied. From the plots of electron density data for the low SZA (orbits 175-205 and 619-650), we have estimated the plasma inhomogeneity inverse scale length $a$ and is found to vary from $10^{-1}-10^{-3} \mathrm{~km}^{-1}$. In regions where the acceleration effect due to Venus gravity and plasma density gradient is in opposite directions, the flute instability would set in when the second term in under-root (equation (7)) becomes more dominant than the first term. For the flute instability to set in, the inequality in terms of oscillation parameter can be written as

$$
a>\left(\omega^{2} / 4 \Omega_{i}^{2}\right)\left(R / \rho_{i}^{2}\right) .
$$

Using the estimated values of $a$, we show the regimes of flute instability in figure 6 . We find that (11) is true for oscillation frequencies $\omega \leqslant 10^{-2} \mathrm{rads}^{-1}$. Oscillations of frequencies higher than $10^{-2} \mathrm{rad} \mathrm{s}^{-1}$ can be excited only under extreme conditions 


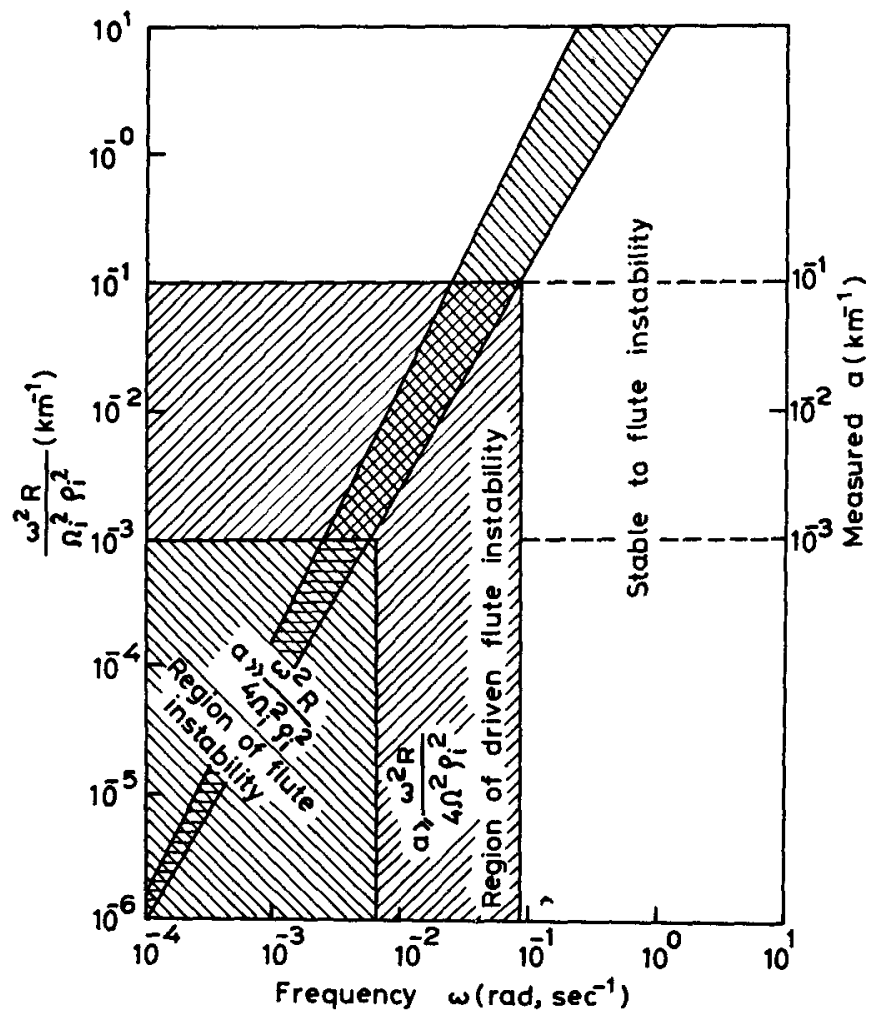

Figure 6. Region of driven flute stability and instability variation with frequencies and other controlling parameter.

for which $a>10^{-1} \mathrm{~km}^{-1}$ or on the other hand variation of parameters which effectively decrease the contribution of the term in (11). It is important to note here that $\omega^{2}$ defined by (11) and appearing in the dispersion equation would change if the Kadomtsev (1965) dispersion equation is considered. The frequency region of instability shown in figure 6 would alter slightly. Such changes may also result due to change of parameters from time to time. The growth rates of the instability have been computed using various measured parameters and their variation with ionopause ion energy as shown in figure 7. The growth rate for larger values of $a$ is found to result in a large growth rate. Therefore, the growth rate corresponding to smaller values of inverse scale length and higher values of magnetic field is relevant only in this study as seen from figure 7. For $\mathrm{O}^{+}$ions of energy $4 \cdot 6 \mathrm{eV}$, the radius of curvature of ionopause and hence the embedded magnetic field becomes equal to the planetary radius. We have shown the growth rate variation with $\mathrm{O}^{+}$energies (velocities) greater than this critical energy for different values of electron density inverse scale length $a$ and the magnetic field strength in the ionopause region. The change of $a$ plays an important role in the growth rate of the flute instability in the ionopause region. The lower magnetic field values in the ionopause region do not affect the growth rate of instability as seen from figure 7 . The growth time of the flute oscillations $\tau=(\operatorname{im} \omega)^{-1}$ is found to vary from $10^{2}-10^{3}$ seconds which compares well with the period of 


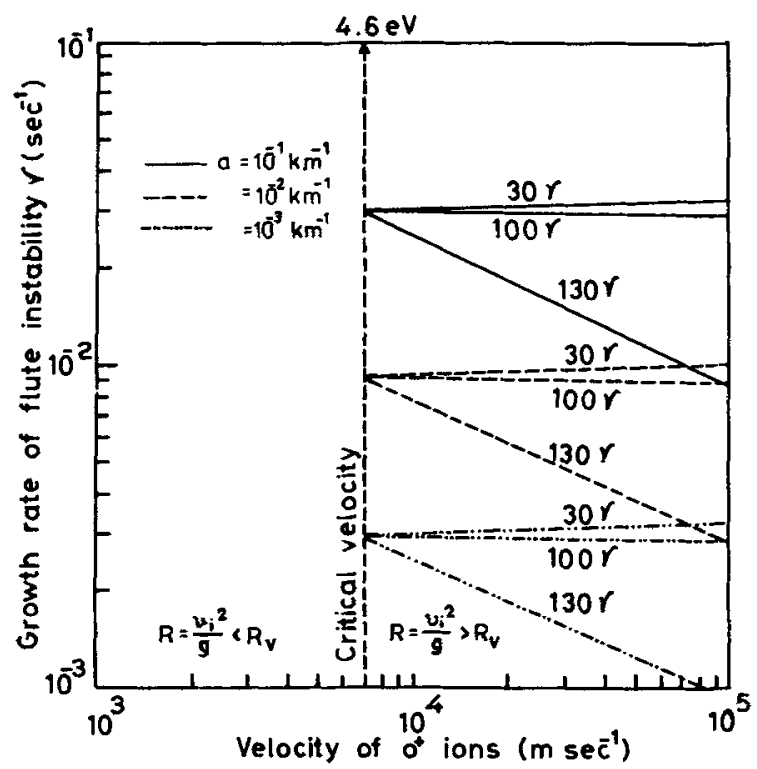

Figure 7. Variation of growth rate of flute instability in the region $V_{i}^{2} / g>R_{\varepsilon}$ with $\mathrm{O}^{+}$ions velocity for different values of Venus magnetic field.

magnetic oscillations observed in the PVO magnetic field data. During certain orbits, the growth time of magnetic oscillations increases especially in the presence of high magnetic field and high energy ions (figure 3). The growth time computed by Elphic and Ershkovich (1984) is somewhat smaller as seen from figure 7.

In certain orbits the bow shock appears closer to the periapsis for low SZA. We find that magnetic oscillations from the bow shock propagate toward the periapsis with decreasing amplitude. These oscillations may at times interact with flute oscillations seen at the ionopause boundary. Such events are small in number and therefore it is not clear how these oscillations would effect the oscillations excited by flute instability. The higher frequency oscillations in the ionopause region far from subsolar region may be triggered by shock front generated singals. These waves are known to be electromagnetic oscillations. The simultaneously recorded electric field by the orbiter's electric field detector (OEFD) also shows copious oscillations.

\section{Conclusions}

The mass-loaded solar wind with magnetic field perpendicular to the solar wind direction is found to be most appropriate for generation of flute instability. The theoretical growth time of flute instability compares well with the magnetic oscillation period observed in the PVO magnetic field data. The flute instability mechanism is more appropriate in the subsolar ionopause region and is overtaken by the Kelvin-Helmholtz instability at higher SZA and in the trans-terminator region of the Venus ionosphere. In this analysis, we have not considered the effect of collisions which may enhance the flute instability in the ionopause region. 


\section{Acknowledgements}

One of us (HOU) is thankful to UGC for a senior research fellowship. Authors thank Professor Chris T Russell for helpful discussions in accomplishing this investigation.

\section{References}

Brace L H, Elphic R C, Curtis S A and Russell C T 1983 Wave structure in the Venus ionosphere down stream of the terminator; Geophys. Res. Lett. $101116-1119$

Cravens T E, Shinagawa H, Nagy A F 1984 The evolution of large scale magnetic fields in the ionosphere of Venus; Geophys. Res. Lett. $11267-270$

Elphic R C and Ershkovich A I 1984 On the stability of the ionopause of Venus; J. Geophys. Res. 89 $997-1002$

Kadomtsev B B 1965 Plasma turbulence (New York: Academic Press)

Luhmann J G, Russell C T and Elphic R C 1984 Time scales for the decay of induced large-scale magnetic fields in the Venus ionosphere; J. Geophys. Res. $89362-368$

Phillips J R, Luhmann J G and Russell C T 1984 Growth and maintenance of magnetic fields in the dayside Venus ionosphere; J. Geophys. Res. 89 10676-10684

Post R F 1969 The physics of high temperature plasma: Matter, properties under unusual conditions (London interscience) pp. 14-266

Rosenbluth M N, Krall N A and Rostoker N 1962 Finite larmor radius stabilization of weakly unstable confined plasma; Nucl. Fusion. Suppl. 1 143-150

Russell C T, Snare R C, Means J D and Elphic R C 1980 Pioneer Venus orbiter flux gate magnetometer; IEEE Trans. Geosci. Remote Sensing GE-18 $32-35$

Russell C T, Singh R N, Luhmann J C, Elphic R C and Brace L H 1987 Waves on subsolar ionopause of Venus; Adv. Space Res. 7(12), 115-118 